\title{
Decreased Aquaporin 4 and 6 mRNAs in Patients With Chronic Otitis Media With Otorrhea
}

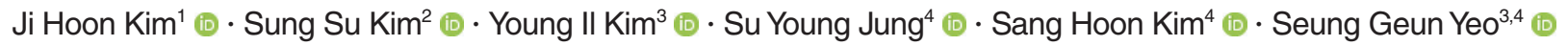 \\ ${ }^{1}$ Department of Otorhinolaryngology-Head and Neck Surgery, Yonsei University Wonju College of Medicine, Wonju; \\ ${ }^{2}$ Department of Biochemistry and Molecular Biology, Medical Science and Engineering Research Center for Bioreaction to Reactive Oxygen \\ Species, BK-21, Kyung Hee University School of Medicine, Seoul; \\ ${ }^{3}$ Medical Science Research Institute, Kyung Hee University Medical Center, Kyung Hee University, Seoul; \\ ${ }^{4}$ Department of Otorhinolaryngology-Head and Neck Surgery, Kyung Hee University School of Medicine, Seoul, Korea
}

Objectives. Aquaporins (AQPs) are integral membrane proteins engaged in the modulation of water homeostasis, but the roles they play in chronic otitis media (COM) have not been well investigated. Accordingly, we undertook document relations between the mRNA expressions of AQPs and COM, and explored the relation between these expressions and otorrhea, which is one of the most common symptoms of COM.

Methods. Levels of the mRNAs of AQP 1, 2, 3, 4, 5, 6, 8, and 10 were assayed by real-time polymerase chain reaction in inflammatory tissue samples from 81 patients with COM with or without otorrhea. Relationships between AQP mRNA levels and the presence or absence of otorrhea, the presence or absence of bacteria, hearing threshold levels, types of hearing loss, and clinical manifestations were also evaluated.

Results. AQP 1, 2, 3, 4, 5, 6, 8, and 10 mRNAs were expressed in inflammatory tissues obtained from all 81 patients with COM with or without otorrhea. AQP 5 mRNA was most expressed in, followed in descending order by AQP 3, 1, 10, $6,8,2$, and 4 . There were no significant intergroup differences in terms of age, sex, duration of illness, levels of hearing loss in both ears, or incidences of conductive or sensorineural hearing loss. However, AQP $4(P=0.035)$ and 6 $(P=0.085)$ mRNA levels were significantly lower in the otorrhea group. In addition, bacteria culture positivity $(P=0.014)$ and the incidence of sensorineural hearing loss $(P=0.020)$ were higher in the otorrhea group.

Conclusion. AQP 1, 2, 3, 4, 5, 6, 8, and 10 are involved in the development of COM. Specifically, it shows reductions in AQP 4 and 6 mRNA levels, as observed in the otorrhea group, have an effect on the clinical manifestations of COM.

Keywords. Aquaporin; Otitis Media; Middle Ear; Chronic; Discharge

\section{INTRODUCTION}

Otitis media $(\mathrm{OM})$ is a generic term for all inflammatory changes originating in the middle ear cavity. OM is encountered in

\footnotetext{
- Received March 30, 2018

Revised October 8, 2018

Accepted October 18, 2018

- Corresponding author: Seung Geun Yeo

Department of Otorhinolaryngology-Head and Neck Surgery, Kyung Hee University School of Medicine, 26 Kyungheedae-ro, Dongdaemun-gu, Seoul 02447, Korea

Tel: +82-2-958-8474, Fax: +82-2-958-8470

E-mail: yeo2park@gmail.com
}

many forms with different onset times and clinical and pathological findings. OM is caused by multiple actions of diverse factors, but mainly develops due to eustachian tube dysfunction or microbial infection. Pathohistological findings in OM include changes in the epithelial cells and subepithelial tissues of the middle ear cavity with osteoclasis and bone resorption of bony structures [1]. More specifically, the major pathohistological findings of chronic otitis media ( $\mathrm{COM})$ include irreversible changes, such as granulation tissue formation, cholesterol granuloma with cholesteatoma, fibrosis, bony destruction, and these changes depend on whether $\mathrm{COM}$ is active or inactive $[2,3]$.

Otorrhea is one of the most common symptoms observed in

Copyright @ 2019 by Korean Society of Otorhinolaryngology-Head and Neck Surgery.

This is an open-access article distributed under the terms of the Creative Commons Attribution Non-Commercial License (http://creativecommons.org/licenses/by-nc/4.0)

which permits unrestricted non-commercial use, distribution, and reproduction in any medium, provided the original work is properly cited. 
COM patients. In most cases, COM is accompanied by intermittent otorrhea, but continuous active otorrhea may occur in perforated OM accompanied by acute infection. Clinical manifestations of otorrhea vary depending on the severity of inflammation $[4,5]$. Furthermore, it has been suggested middle ear effusion and ejection dysfunction derived from OM might be associated with aquaporin (AQP) dysfunctions, which are known to contribute to the control of water homeostasis [6]. AQP expressions in the middle ear and eustachian tube in the presence of OM has been investigated in animals and humans [7-11], but no study has yet addressed AQP expression levels in COM patients or the role of AQPs in relation to COM.Accordingly, in the present study, we explored whether AQP 1, 2, 3, 4, 5, 6, 8, and 10 mRNAs are considered to be related to OM, are expressed in tissues of the middle ear cavity in COM, and whether their expressions are related to clinical manifestations. In addition, we also investigated the relation between AQP expression and the development of otorrhea.

\section{MATERIALS AND METHODS}

\section{Subjects}

The study subjects were 81 patients who visited the Department of Otorhinolaryngology-Head and Neck Clinic of Kyung Hee University Medical Center from May 2015 to August 2017. Patients who visited the center for symptoms such as hearing loss, otorrhea, tinnitus, otalgia, or dizziness of more than 3 months and diagnosed with a perforated tympanic membrane (TM) with or without otorrhea, those with abnormal lesions in the middle ear and mastoid cavity as determined by temporal bone computed tomography, and those with granulation tissues and chronic inflammation based on biopsy finding conducted during surgery, were defined as COM patients. Particular attention was paid to the identification of the origin of otorrhea development in TMs or external auditory canals using otoscope or endoscope and by referring to patient complaints. In patients with hearing loss, pure tone audiometry (PTA) was performed to measure hearing thresholds, and bacterial culture tests were conducted for patients with otorrhea. Before study commencement, written informed consent was obtained from patients, parents, or

\section{H I G G H L I I G H T S S}

- Aquaporin (AQP) 1, 2, 3, 4, 5, 6, 8, and 10 mRNAs were expressed in inflammatory tissues obtained from all 81 patients with chronic otitis media with or without otorrhea.

- AQP $4(P=0.035)$ and $6(P=0.085)$ mRNA levels were significantly lower in the otorrhea group.

- AQP 4 and 6 mRNAs reduction have an effect on the clinical manifestations of chronic otitis media. guardians with respect to the use patient samples after providing an explanation of study purposes. Patients suspected of having acute otitis media (AOM), head or neck anomaly, systemic disease, or congenital or acquired immune deficiencies were excluded. This cross-sectional study was approved by the Institutional Review Board of Kyung Hee University Medical Center (IRB No. 2017-12-030).

\section{Bacterial culture}

When otorrhea was detected in COM patients, relevant samples were collected and bacterial culture was performed. After cleaning the external auditory canal, otorrhea samples were collected under aseptic conditions using sterilized cottons and an antiseptic otoscope to prevent contact with the external auditory canal. All clinical samples were collected using sterile cotton swabs (Xomed Trace Products, Jacksonville, FL, USA), which were immediately immersed in Stuart transport medium. Solid blood agar and liquid thioglycollate medium (Hangang, Gunpo, Korea) were inoculated with these swabs and then incubated for 24 hours at $35.8^{\circ} \mathrm{C}$. Colony-forming bacteria were identified by Gram stain and biochemical tests.

\section{RNA extraction and real-time PCR}

Total RNA was purified from patient tissue samples using TRIzol solution, according to the manufacturer's instructions (Invitrogen, Carlsbad, CA, USA). RNAs were isolated using a RNeasy mini kit (Qiagen, Hilden, Germany). First-strand complementary DNA (cDNA) synthesis was performed with $1 \mu \mathrm{g}$ of total RNA, which was transcribed to cDNA using a reverse transcription system and random hexamers (Promega, Madison, WI, USA), according to the manufacturer's instructions. To obtain primer

Table 1. Primer sequences used for real-time PCR

\begin{tabular}{lllc}
\hline Name & Direction & \multicolumn{1}{c}{ Sequence } & $\begin{array}{c}\text { Product } \\
\text { size (bp) }\end{array}$ \\
\hline AQP1 & Forward & 5'-TGGACACCTCCTGGCTATTG-3' & 164 \\
& Reverse & 5'-GGGCCAGGATGAAGTCGTAG-3' & \\
AQP2 & Forward & 5'-CACCCCTGCTCTCTCCATA-3' & 139 \\
& Reverse & 5'-GAAGACCCAGTGGTCATCAAAT-3' & \\
AQP3 & Forward & 5'-TCAATGGCTTCTTGACCAGTTCA-3' & 389 \\
& Reverse & 5'-CTTCACATGGGCCAGCTTCACATT-3' & \\
AQP4 & Forward & 5'-GAAGGCATGAGTGACAGACC-3' & 130 \\
& Reverse & 5'-ATTCCGCTGTGACTGCTTC-3' & \\
AQP5 & Forward & 5'-GCCACCTTGTCGGAATCTAC-3' & 148 \\
& Reverse & 5'-TAAAGGATGGCAGCCAGGAC-3' & \\
AQP6 & Forward & 5'-CACCTCATTGGGATCCACTT-3' & 147 \\
& Reverse & 5'-GTTGTAGATCAGTGAGGCCA-3' & \\
AQP8 & Forward & 5'-GTGCCTGTCGGTCATTGAG-3' & 125 \\
& Reverse & 5'-CAGGGTTGAAGTGCCACC-3' & \\
AQP10 & Forward & 5'-GCCTGGGAACAACAGTCATT-3' & 155 \\
& Reverse & 5'-TGAGAAGCCTGAGTCCTGGT-3' & \\
B-Actin & Forward & 5'-GCGAGAAGATGACCCAGATC-3' & 77 \\
& Reverse & 5'-GGATAGCACAGCCTGGATAG-3' & \\
\hline
\end{tabular}

$\mathrm{PCR}$, polymerase chain reaction; $\mathrm{AQP}$, aquaporin. 
sequences, we referred to previous studies (Table 1) [6-11]. Realtime PCR was performed using the StepOnePlus real-time PCR system and Power SYBR Green PCR Master Mix (Applied Biosystems, Foster City, CA, USA). PCR reactions were performed with $1 \mu \mathrm{L}$ of cDNA in a $20 \mu \mathrm{L}$ reaction mixture containing 10 $\mu \mathrm{L}$ of Power SYBR Green PCR Master Mix, $2 \mu \mathrm{L}$ of primers, and $7 \mu \mathrm{L}$ of PCR-grade water. Initial denaturation was conducted at $95^{\circ} \mathrm{C}$ for 10 minutes and was followed by 40 amplification cycles of $95^{\circ} \mathrm{C}$ for 15 seconds and $60^{\circ} \mathrm{C}$ for 1 minute. The crossing points of target genes with $\beta$-actin were calculated using the formula $2^{\text {-(target gene- } \beta \text {-actin) }}$ and the natural logarithm was used in all logarithmic transformations. Real-time PCR was performed twice and their average value was obtained.

\section{Evaluation of hearing level and type of hearing loss}

PTA was performed by measuring air conduction (AC) and bone conduction $(\mathrm{BC})$, and hearing levels were calculated as the average of three frequencies $(500,1,000$, and 2,000 Hz). Based on PTA results, types of hearing loss were classified as either conductive hearing loss (CHL) or sensorineural hearing loss (SNHL). Patients with decreased AC and normal BC, an airbone gap $(\mathrm{ABG})>10 \mathrm{~dB} \mathrm{HL}$, and a negative Rinne test were diagnosed as having $\mathrm{CHL}$; whereas patients with similar decreases in $\mathrm{AC}$ and $\mathrm{BC}$, or $\mathrm{ABG} \leq 10 \mathrm{~dB} \mathrm{HL}$, and with a positive Rinne test were diagnosed as having SNHL [12].

\section{Statistical analysis}

The analysis was conducted using IBM SPSS ver. 20.0 (IBM Corp., Armonk, NY, USA). Results are expressed using descriptive statistics as means and standard deviations, frequencies, or percentages. The Kolmogorov-Smirnov test was used to determine numerical variables were normally distributed. The significances of intergroup differences were determined using the stu-

Table 2. Demographic and clinical characteristics according to presence or absence of otorrhea in COM patients

\begin{tabular}{lccc}
\hline Variable & $\begin{array}{c}\text { COM with } \\
\text { otorrhea }(n=53)\end{array}$ & $\begin{array}{c}\text { COM without } \\
\text { otorrhea }(n=28)\end{array}$ & $P$-value \\
\hline Age $(\mathrm{yr})$ & $55.5 \pm 12.7$ & $57.5 \pm 16.2$ & 0.536 \\
Male:female & $23(43.4): 30(56.6)$ & $9(32.1): 19(67.9)$ & 0.331 \\
Disease onset (mo) & $54.9 \pm 106.9$ & $43.9 \pm 60.5$ & 0.187 \\
Affected side, & $24: 18: 11$ & $11: 10: 7$ & 0.729 \\
Rt:Lt:both & $(45.3: 34.0: 20.8)$ & $(39.3: 35.7: 25.0)$ & \\
Presence of bacteria & $23(43.4)$ & $5(17.9)$ & $0.014^{*}$ \\
Type of healing loss, & $30(56.6): 23(43.4)$ & $22(78.6): 6(21.4)$ & $0.020^{*}$ \\
CHL:SNHL & & & \\
PTA, AC (dB) & $56.5 \pm 22.1$ & $54.1 \pm 20.4$ & 0.627 \\
PTA, BC (dB) & $27.9 \pm 17.9$ & $26.0 \pm 17.6$ & 0.640 \\
\hline
\end{tabular}

Values are presented as mean \pm standard deviation or number (\%). $\mathrm{COM}$, chronic otitis media; Rt, right; Lt, left; $\mathrm{CHL}$, conductive hearing loss; SNHL, sensorineural hearing loss; PTA, pure tone audiometry; AC, air conduction; $\mathrm{BC}$, bone conduction.

${ }^{\star} P<0.05$. dent $t$-test, the Mann-Whitney $U$-test, or the Kruskal-Wallis test followed by post hoc comparisons (Tukey's honest significant difference test and the Bonferroni-adjusted Mann-Whitney test). Statistical significance was accepted for $P$-values $<0.05$.

\section{RESULTS}

\section{Demographic and clinical characteristics}

Eighty-one patients diagnosed with COM were enrolled in this study. No differences were found between the otorrhea and non- otorrhea groups with regard to age, sex, duration of illness, affected side, or levels of hearing loss $(P>0.05)$. However, the rate of SNHL was higher in the otorrhea group $(P=0.020)$ (Table 2).

Comparison of the mRNA expressions of AQP 1, 2, 3, 4, 5, 6, 8 , and 10

AQP 1, 2, 3, 4, 5, 6, 8, and 10 mRNAs were expressed in inflammatory tissues obtained from the 81 study subjects. AQP 5 mRNA was most predominantly expressed, followed by AQP 3, $1,10,6,8,2$, and 4 in decreasing order (Table 3).

Table 3. Comparison of expression of AQP 1, 2, 3, 4, 5, 6, 8, and 10 mRNAs

\begin{tabular}{lc}
\hline Type of AQP & Relative mRNA expression level \\
\hline AQP 1 & $-4.044 \pm 2.145$ \\
AQP 2 & $-5.828 \pm 4.463$ \\
AQP 3 & $-3.623 \pm 4.367$ \\
AQP 4 & $-6.685 \pm 2.617$ \\
AQP 5 & $-3.492 \pm 2.075$ \\
AQP 6 & $-5.695 \pm 3.133$ \\
AQP 8 & $-6.765 \pm 2.444$ \\
AQP 10 & $-4.514 \pm 3.107$ \\
\hline
\end{tabular}

Values are presented as mean \pm standard deviation.

$\mathrm{AQP}$, aquaporin.

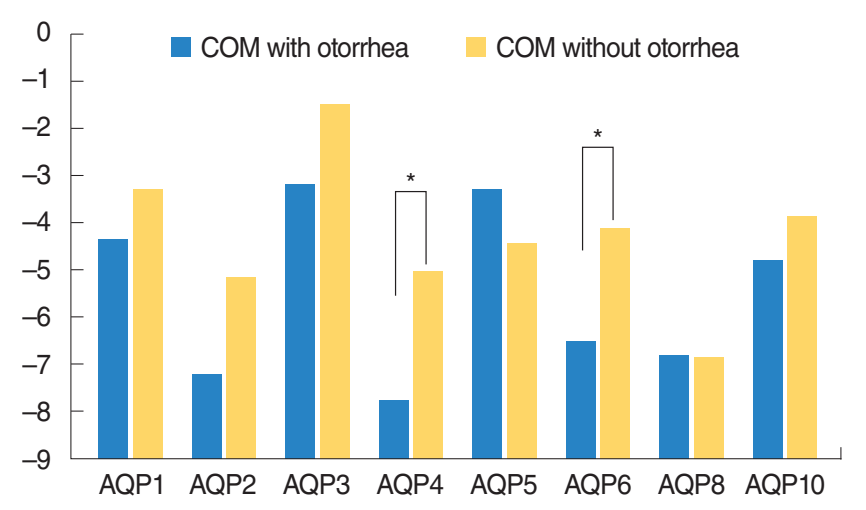

Fig. 1. Comparison of expression of AQP1, 2, 3, 4, 5, 6, 8, and 10 mRNAs according to presence or absence of otorrhea in COM patients. COM, chronic otitis media; AQP, aquaporin. ${ }^{\star} P<0.05$ 
Table 4. Comparison of expression of AQP1, 2, 3, 4, 5, 6, 8, and 10 mRNAs according to types of hearing loss

\begin{tabular}{lllc}
\hline Type of AQP & $\mathrm{CHL}(\mathrm{n}=52)$ & $\mathrm{SNHL}(\mathrm{n}=29)$ & $P$-value \\
\hline AQP 1 & $-3.748 \pm 2.206$ & $-4.554 \pm 1.931$ & 0.196 \\
AQP 2 & $-4.809 \pm 4.531$ & $-7.874 \pm 3.601$ & 0.397 \\
AQP 3 & $-2.584 \pm 4.205$ & $-5.292 \pm 4.255$ & 0.137 \\
AQP 4 & $-5.923 \pm 3.089$ & $-7.600 \pm 3.334$ & 0.383 \\
AQP 5 & $-3.754 \pm 2.272$ & $-3.065 \pm 1.742$ & 0.251 \\
AQP 6 & $-4.989 \pm 3.314$ & $-7.082 \pm 2.195$ & 0.387 \\
AQP 8 & $-6.813 \pm 2.290$ & $-8.080 \pm 2.573$ & 0.447 \\
AQP 10 & $-3.967 \pm 3.253$ & $-5.671 \pm 2.565$ & 0.377 \\
\hline
\end{tabular}

Values are presented as mean \pm standard deviation.

$\mathrm{AQP}$, aquaporin; $\mathrm{CHL}$, conductive hearing loss; $\mathrm{SNHL}$, sensorineural hearing loss.

Table 5. Comparison of expression of AQP1, 2, 3, 4, 5, 6, 8, and 10 $\mathrm{mRNAs}$ according to severity of hearing loss

\begin{tabular}{llll}
\hline \multirow{2}{*}{ Type of AQP } & \multicolumn{2}{c}{ PTA } & P-value \\
\cline { 2 - 3 } & $\leq 40 \mathrm{~dB}(n=23)$ & $>40 \mathrm{~dB}(\mathrm{n}=58)$ & \\
\hline AQP 1 & $-3.604 \pm 2.627$ & $-4.341 \pm 1.844$ & 0.238 \\
AQP 2 & $-7.374 \pm 3.567$ & $-4.773 \pm 4.439$ & 0.687 \\
AQP 3 & $-2.686 \pm 4.385$ & $-4.018 \pm 4.307$ & 0.494 \\
AQP 4 & $-6.655 \pm 2.426$ & $-6.711 \pm 2.926$ & 0.436 \\
AQP 5 & $-3.710 \pm 2.211$ & $-3.432 \pm 2.203$ & 0.285 \\
AQP 6 & $-5.029 \pm 3.494$ & $-6.065 \pm 3.020$ & 0.600 \\
AQP 8 & $-7.923 \pm 2.531$ & $-5.793 \pm 1.892$ & 0.458 \\
AQP 10 & $-4.146 \pm 3.073$ & $-4.694 \pm 3.243$ & 0.420 \\
\hline
\end{tabular}

Values are presented as mean \pm standard deviation.

AQP, aquaporin; PTA, pure tone audiometry.

Comparison of the mRNA expressions of AQP 1, 2, 3, 4, 5, 6, 8 , and 10 mRNAs in the two study groups

AQP expressions were noticeably lower in the otorrhea group than in the non-otorrhea group, and the expressions of AQP 4 and 6 mRNAs were significantly lower $(P<0.05)$ (Fig. 1).

Relations between the mRNA expressions of AQP 1, 2, 3, 4, 5, 6,8 , and 10 and hearing loss type and severity

The 81 study subjects were divided into CHL and SNHL groups based on the results of PTA. AQP expressions were found to be higher in the CHL group than in the SNHL group, but not significantly so $(P>0.05)$ (Table 4$)$. Similarly, the study subjects were divided into $\leq 40 \mathrm{~dB}$ and $>40 \mathrm{~dB}$ groups based on serviceable hearing levels. There were no differences of AQP expressions in these two groups $(P>0.05)$ (Table 5).

\section{DISCUSSION}

The incidence of $\mathrm{OM}$ is very high and the prevalence of $\mathrm{COM}$ is dependent on endogenic and exogenic factors. The former includes genetic, racial, and sex- and age-related factors, and the latter environmental, social, and cultural factors. In most cases,
Table 6. The studies of expression of AQPs in middle ear and eustachian tube

\begin{tabular}{|c|c|c|c|c|c|}
\hline \multirow{2}{*}{ AQP } & \multicolumn{2}{|c|}{ Tissue type } & \multicolumn{2}{|c|}{ Detection method } & \multirow{2}{*}{ Study } \\
\hline & NHMEE OME-M & OME-E & $\mathbb{H C}$ & qPCR & \\
\hline 5 & 0 & & & 0 & Samuel et al. [17] \\
\hline 3,10 & & 0 & & 0 & Jin et al. [11] \\
\hline $\begin{array}{c}1,2,3,4,5,6 \\
8,10,11\end{array}$ & 0 & & & 0 & Seo and Choi [18] \\
\hline $1,3,5$ & 0 & & 0 & & Seo and Choi [18] \\
\hline
\end{tabular}

AQP, aquaporin; NHMEE, normal human middle ear epithelium; OME-M, mucosa of middle ear cavity from patients with otitis media with effusion; OME-E, effusion of middle ear cavity from patients with otitis media with effusion; IHC, immunohistochemistry; qPCR, quantitative real-time polymerase chain reaction.

AOM can be cured without after effects, but in some recurrent $\mathrm{OM}$ or OM with effusion (OME) inflammation persists or reoccurs. Furthermore, even after treatment, AOM can become chronic due to incomplete cure of inflammation in the middle ear cavity. However, the factors responsible for the development of chronic inflammation after acute infections of the middle ear and mastoid cavity have not been elucidated [1-5].

In a recent study, it was concluded water and electrolyte channel proteins in the middle ear mucous membrane contribute to the generation or elimination of fluid in the middle ear [6]. With regard to the defensive role of mucous epithelium of the middle ear and eustachian tube, homeostasis of mucosal and pericilliary fluid layers produced by ciliated and goblet cells should be maintained, and to achieve this water must be appropriately controlled [13]. Changes in the amount of water in the middle ear mucous membrane and in electrolyte compositions can be attributed to causes of middle ear diseases, such as, OM, chronicization, middle ear effusion, and otorrhea, and AQPs are engaged in this modulation of water levels $[14,15]$.

It has been reported AQPs are involved in urine concentration, the control of water egress in sweat, ischemia, tumors, edema (caused by viral infection), and during cell migration, metabolic processes, and the transmission of neural information. The AQPs that move only water are AQP $0,1,2,4,5,6$, and 8.AQP 3, 7, 9, and 10 are called aquaglycoporins as they move glycerol and water, and eight AQP types, that is, AQP 1-6, 8, and 10, have been reported to be expressed in the middle ears of humans and animals [11,16-19]. Previous studies conducted on human subjects have reported the presence of AQP 5 mRNA in the mucous membranes of OME, AQPs 3 and 10 in the effusion of OME, and AQPs 1, 2, 3, 4, 5, 6, 8, 10, and 11 in normal human middle ear epithelium (Table 6). However, no study has previously determined whether the mRNAs of AQP 1, 2, 3, 4, 5, 6, 8, 10, and 11 are expressed in COM patients. In the present study, we examined relations between the expressions of the mRNAs of AQP16,8 , and 10 and the pathophysiology of COM. We found that the mRNAs of AQP 1-6, 8, and 10 were expressed in the granulation tissues of $\mathrm{COM}$ patients. These expressions, in decreasing 
order, were as follows; AQP $5>3>1>10>6>8>2>4$. In a study using murine COM models it was reported the expressions of AQP mRNAs in middle ears were 2.79-fold higher in uninfected ears and 5.40-fold higher in infected ears than in normal controls, and thus, it was suggested inflammation contributes to the upregulations of AQPs [20]. Similarly, other studies have shown that the mRNAs of AQP 1, 2, 3, 4, 5, 6, 8, 10, and 11 mRNAs are expressed in OME patients. Collectively, these results show inflammation in the middle ear affects the mRNA expressions of $\mathrm{AQPs}$, and that these AQPs play the similar roles in immune response in patients with COM.

Changes in the expression and location of AQPs under specific conditions are associated with many diseases. Furthermore, malfunctions in the processes responsible for the metabolisms of AQPs can cause various diseases. Specifically, a number of studies have indicated that tumors develop and grow more slowly in AQP 1 knockout mice [21], skin hydration, elasticity, barrier recovery, and wound healing are reduced in AQP 3 knockout mice [22], migrations of stellate cells are reduced by chemotactic factors, and glial scar formation engaged in restoring injured cerebral tissues was delayed in AQP 4 knockout mice [23], and sensitivities to light and sound are attenuated in AQP 4 knockout mice [24]. Interestingly, it has also been shown adipocyte hypertrophy and fat accumulation in AQP 7 knockout mice [25].

AQPs are expressed in immune cells of the innate and adaptive immune systems in human. If pathogens penetrate and damage the middle ear, ion channels in relevant cells are disrupted, and consequently the functions and expressions of transmembrane AQPs are altered. Such conditions cause abnormal changes in intracellular signals and cell membrane potentials resulting in exudation [26]. In the present study, it was found that the mRNA expressions of all AQPs were lower in the otorrhea group than in the non-otorrhea group, and those of AQP 4 and 6 were significantly lower. These results are similar to those obtained in other disease conditions and it has been established dysfunctional AQP expressions disrupt the modulations of water and inflammation. Accordingly, our observations suggest that $\mathrm{AQPs}$ play a role in the regulation of water levels in the tissues of the middle ear, and that disruption of water homeostasis in middle ear cells due to diminished AQP expression results in the excessive accumulation of water in the middle ear cavity, and thus, persistent otorrhea.

Generally, COM is accompanied by hearing loss, usually CHL, although prolonged or severe inflammation may be accompanied by labyrinthitis, which leads to mixed hearing loss or SNHL. Level of hearing loss are determined by the sizes and locations of TM perforations and the states and motilities of ossicular chains [27]. Furthermore, ossicular lesions are dependent on the type of COM and the presence or absence of otorrhea. In other words, ossicular lesions are encountered more frequently in active COM with otorrhea than in inactive COM without otorrhea [28]. However, the relation between hearing loss and AQPs have not been explored in animals or humans. In the present study, we compared the expressions of AQP mRNAs with respect to hearing loss type and level, to determine whether AQP expressions are dependent on the level, type, and presence of hearing loss and whether the presence of SNHL is dependent on the expressions of specific AQP mRNAs. However, contrary to our expectations, the results obtained were not statistically significant.

The present study has several limitations that warrant mention. First, for ethical reasons, we were not able to incorporate healthy ears as controls. Second, we measured AQP mRNA levels and not protein levels, and thus, we are unable to comment on the expressions of AQP proteins. Third, in the patients who were classified as non-otorrhea group because of the absence of otorrhea in external auditory canals, inflammation and otorrhea were found in the middle ears and mastoid cavity during surgery. Fourth, antibiotics had been administered before surgery to members of the both otorrhea and non-otorrhea groups, and this may have altered the mRNA expressions of AQPs.

In conclusion, the present study shows the mRNAs of AQP 1 , $2,3,4,5,6,8$, and 10 are associated with the development of $\mathrm{COM}$, and that in patients with COM and otorrhea, the mRNA expressions of AQP 4 and 6 are significantly decreased. Our results suggest the mRNA expressions of AQPs influence the development of otorrhea in COM patients.

\section{CONFLICT OF INTEREST}

No potential conflict of interest relevant to this article was reported.

\section{ACKNOWLEDGMENTS}

This work was supported by the National Research Foundation of Korea (NRF) grant funded by the Korea Government (MSIP) (No. 2011-0030072), Republic of Korea.

\section{ORCID}

$\begin{array}{ll}\text { Ji Hoon Kim } & \text { https://orcid.org/0000-0002-0844-3047 } \\ \text { Sung Su Kim } & \text { https://orcid.org/0000-0002-6817-7668 } \\ \text { Young Il Kim } & \text { https://orcid.org/0000-0002-4178-8119 } \\ \text { Su Young Jung } & \text { https://orcid.org/0000-0001-6398-1297 } \\ \text { Sang Hoon Kim } & \text { https://orcid.org/0000-0001-5045-5060 } \\ \text { Seung Geun Yeo } & \text { https://orcid.org/0000-0001-8021-1024 }\end{array}$

\section{AUTHOR CONTRIBUTIONS}

Conceptualization: SGY. Data curation: SYJ, SHK. Formal anal- 
ysis: SYJ, SHK. Funding acquisition: SGY. Methodology: SSK, YIK, SYJ, SHK. Project administration: JHK, SGY. Visualization: SSK, SGY.Writing - original draft: JHK, SGY.Writing - review \& editing: all authors.

\section{REFERENCES}

1. Kim CS, Jung HW, Yoo KY. Prevalence and risk factors of chronic otitis media in Korea: results of a nation-wide survey. Acta Otolaryngol. 1993 May;113(3):369-75.

2. Paparella MM, Schachern PA, Cureoglu S. Chronic silent otitis media. ORL J Otorhinolaryngol Relat Spec. 2002 Mar-Apr;64(2):65-72.

3. da Costa SS, Paparella MM, Schachern PA, Yoon TH, Kimberley BP. Temporal bone histopathology in chronically infected ears with intact and perforated tympanic membranes. Laryngoscope. 1992 Nov; 102(11):1229-36.

4. Meyerhoff WL, Kim CS, Paparella MM. Pathology of chronic otitis media. Ann Otol Rhinol Laryngol. 1978 Nov-Dec;87(6 Pt 1):749-60.

5. Mauk GW, Behrens TR. Historical, political, and technological context associated with early identification of hearing loss. Semin Hear. 1993 Feb;14(1):1-17.

6. Agre P. The aquaporin water channels. Proc Am Thorac Soc. 2006 Mar;3(1):5-13.

7. Kang SH, Chang KH, Ohcho S, Lee HY, Cha K, Moon SK, et al. Expression of water channel proteins (aquaporins) in the rat Eustachian tube and middle ear mucosa. Acta Otolaryngol. 2007 Jul;127(7):687-92.

8. Borgnia M, Nielsen S, Engel A, Agre P. Cellular and molecular biology of the aquaporin water channels. Annu Rev Biochem. 1999;68: 425-58.

9. Zhang Q, Liu C, Gao X, HuY, GuoW, Sun J, et al. Expression pattern of aquaporin 1 in the middle ear of the guinea pig with secretory otitis media. ORL J Otorhinolaryngol Relat Spec. 2009 Apr;71(2): 70-7.

10. Zhang Q, Liu C, Wang J, Sun J, Hu Y, Chen G, et al. Expression pattern of aquaporin 4 and 5 in the middle ear of guinea pigs with secretory otitis media. Acta Otolaryngol. 2010 Jan;130(1):68-74.

11. Jin Z, Cha SH, Choi YS, Kim YI, Choi SA, Yeo SG. Expression of CXCL4 and aquaporin 3 and 10 mRNAs in patients with otitis media with effusion. Int J Pediatr Otorhinolaryngol. 2016 Feb;81:33-7.

12. Rovers MM, Schilder AG, Zielhuis GA, Rosenfeld RM. Otitis media. Lancet. 2004 Feb;363(9407):465-73.

13. Sasai M, Yamamoto M. Pathogen recognition receptors: ligands and signaling pathways by Toll-like receptors. Int Rev Immunol. 2013 Apr;
32(2):116-33.

14. Juhn S, Paparella MM, Goycoolea MV, Kim CS, Giebink S. Pathogenesis of otitis media. Ann Otol Rhinol Laryngol. 1977 Jul;86(4): 481-92.

15. Paparella MM, Schachern PA, YoonTH,Abdelhammid MM, Sahni R, da Costa SS. Otopathologic correlates of the continuum of otitis media. Ann Otol Rhinol Laryngol Suppl. 1990 Jun;148:17-22.

16. Ishibashi K, Morishita Y,Tanaka Y.The evolutionary aspects of aquaporin family. Adv Exp Med Biol. 2017 Mar;969:35-50.

17. Samuels TL, Yan JC, Khampang P, Dettmar PW, MacKinnon A, Hong $\mathrm{W}$, et al. Association of gel-forming mucins and aquaporin gene expression with hearing loss, effusion viscosity, and inflammation in otitis media with effusion. JAMA Otolaryngol Head Neck Surg. 2017 Aug;143(8):810-7.

18. Seo YJ, Choi JY. Expression and localization of aquaporin water channels in human middle ear epithelium. Otol Neurotol. 2015 Aug; 36(7):1284-9.

19. MacArthur CJ, Hausman F, Kempton JB, Trune DR. Murine middle ear inflammation and ion homeostasis gene expression. Otol Neurotol. 2011 Apr;32(3):508-15.

20. Saadoun S, Papadopoulos MC, Hara-Chikuma M, Verkman AS. Impairment of angiogenesis and cell migration by targeted aquaporin-1 gene disruption. Nature. 2005 Apr;434(7034):786-92.

21. Hara M, Ma T, Verkman AS. Selectively reduced glycerol in skin of aquaporin-3-deficient mice may account for impaired skin hydration, elasticity, and barrier recovery. J Biol Chem. 2002 Nov;277(48): 46616-21.

22. Saadoun S, Papadopoulos MC, Watanabe H, Yan D, Manley GT, Verkman AS. Involvement of aquaporin-4 in astroglial cell migration and glial scar formation. J Cell Sci. 2005 Dec;118(Pt 24):5691-8.

23. Da T, Verkman AS. Aquaporin-4 gene disruption in mice protects against impaired retinal function and cell death after ischemia. Invest Ophthalmol Vis Sci. 2004 Dec;45(12):4477-83.

24. Hara-Chikuma M, Sohara E, Rai T, Ikawa M, Okabe M, Sasaki S, et al. Progressive adipocyte hypertrophy in aquaporin-7-deficient mice: adipocyte glycerol permeability as a novel regulator of fat accumulation. J Biol Chem. 2005 Apr;280(16):15493-6.

25. Amiry-Moghaddam M, Ottersen OP. The molecular basis of water transport in the brain. Nat Rev Neurosci. 2003 Dec;4(12):991-1001.

26. Karja J, Jokinen K, Seppala A. Destruction of ossicles in chronic otitis media. J Laryngol Otol. 1976 Jun;90(6):509-18.

27. Sade J, Berco E, Buyanover D, Brown M. Ossicular damage in chronic middle ear inflammation. Acta Otolaryngol. 1981 Sep-Oct; 92(3-4):273-83.

28. Gonen T, Walz T. The structure of aquaporins. Q Rev Biophys. 2006 Nov;39(4):361-96. 